seven loss-of-function variants, 22 missense variants and two in-frame indels. Sanger sequencing of the unaffected parents of seven cases identified five de novo variants. Three NOTCH1 variants (p.G200R, p.C607Y and p.N1875S) were subjected to functional evaluation and two showed a reduction in Jagged1-induced NOTCH signalling. FLT4 variants were found in 2.4\% (95\% CI:1.6-3.8\%) of TOF patients, with 21 patients harbouring 22 unique, deleterious variants. The variants identified were distinct to those that cause the congenital lymphoedema syndrome Milroy disease. In addition to NOTCH1, FLT4 and the well-established TOF gene, TBX1, we identified potential association with variants in several other biologically plausible candidate genes. In summary, the NOTCH1 locus is the most frequent site of genetic variants predisposing to non-syndromic TOF, followed by FLT4. Together, variants in these genes are found in almost $7 \%$ of TOF patients.

\section{STRESS MYOCARDIAL OXYGENATION AND NOT PERFUSION RESERVE DETERMINES ARRHYTHMIC RISK IN HYPERTROPHIC CARDIOMYOPATHY: INSIGHTS FROM A NOVEL OXYGEN-SENSITIVE CMR APPROACH}

${ }^{1}$ Betty Raman** ${ }^{1}$ Kenneth Chan, ${ }^{1}$ Masliza Mahmod, ${ }^{1}$ Moritz Hundertmark, ${ }^{1}$ Rina Ariga, ${ }^{1}$ Sanjay Sivalokanathan, ${ }^{2}$ Theodoros Karamitsos, ${ }^{3}$ Joseph Selvanayagam, ${ }^{1}$ Aaron Hess, ${ }^{1}$ Elizabeth M Tunnicliffe, ${ }^{1}$ Hugh Watkins, ${ }^{1}$ Stefan Neubauer*. ${ }^{1}$ Division of Cardiovascular Medicine, Radcliffe Department of Medicine, University of Oxford, Oxford, UK; ${ }^{2}$ First Department of Cardiology, Aristotle University of Thessaloniki, AHEPA Hospital, Thessaloniki, Greece; ${ }^{3}$ Department of Cardiovascular Medicine, Flinders Medical Centre, Bedford Park, Adelaide. Australia

10.1136/heartjnl-2019-BCS.227

Myocardial ischemia has long been implicated in promoting arrhythmic events and triggering sudden cardiac death in hypertrophic cardiomyopathy (HCM). However, the evidence for this is scarce due to challenges presented in direct ischemia assessment which generally requires an invasive approach. Blood oxygen level dependent cardiac magnetic resonance (BOLD CMR) permits the non-invasive assessment of tissue oxygenation, without gadolinium contrast, overcoming many limitations suffered by traditional methods. From a clinical perspective, T2-prepared steady-state free precession (T2-SSFP) BOLD is promising, but suffers from reduced diagnostic accuracy, owing to imprecisions in BOLD measurements secondary to heart-rate (HR) dependence. To resolve this, we developed a novel oxygen-sensitive CMR approach, Fast Low Angle Shot (FLASH) interleaved T2-SSFP BOLD, which was designed to eliminate both HR dependence and spatial variations seen with standard T2-SSFP BOLD. A comparison of both standard and novel approaches in 20 healthy subjects confirmed that FLASH-normalised T2-SSFP BOLD is highly reproducible, HR independent and more precise than standard T2-SSFP BOLD. In addition, the mean BOLD effect did not differ between the two methods. Importantly, using this novel approach, one could visualise changes in oxygen-sensitive signal from rest to stress qualitatively, making it feasible for direct incorporation into a clinical work flow. We then set out to test the hypothesis that stress oxygenation (as assessed on FLASH normalised T2-SSFP BOLD) is more powerful that myocardial perfusion reserve (MPRI) at determining arrhythmic risk in HCM patients. Adenosine stress BOLD, first pass perfusion imaging and late gadolinium enhancement CMR were undertaken in 103 genotyped-HCM patients. All patients underwent 24-hour Holter to monitor for evidence of ventricular tachycardia $(\geq 3$ beats, $\geq 120$ beats per minute). Thirty-two age- and sexmatched healthy subjects served as controls. Although both stress oxygenation and MPRI were impaired in HCM, only stress oxygenation, but not MPRI, associated with ventricular tachycardia on univariate analysis. There was a step-wise increase in ventricular tachycardia prevalence with decreasing quartiles of stress oxygenation. HCM patients with the lowest quartile of oxygenation were at a three-fold increased risk of ventricular tachycardia (OR 3.04, $\mathrm{p}=0.04$ ) after adjusting for LGE mass, age and baseline risk of sudden cardiac death. Sarcomeric mutation status was an independent determinant of stress oxygenation, irrespective of the extent of hypertrophy, MPRI or LGE burden (univariate predictors). In conclusion, we have successfully developed and implemented a novel oxygen-sensitive CMR method which has provided important insights into the role of stress oxygenation as a promising biomarker of arrythmic risk and potential therapeutic target for drug discovery in HCM. 